\title{
A New Visualization Approach to Re-Contextualize Indigenous Knowledge in Rural Africa
}

\author{
Kasper Rodil ${ }^{1}$, Heike Winschiers-Theophilus ${ }^{2}$, Nicola J. Bidwell ${ }^{3,4}$, Søren Eskildsen ${ }^{1}$, \\ Matthias Rehm ${ }^{1}$, and Gereon Koch Kapuire ${ }^{2}$ \\ ${ }^{1}$ Department of Architecture, Design, and Media Technology, Aalborg University, Denmark \\ ${ }^{2}$ School of Information Technology, Polytechnic of Namibia, Namibia \\ ${ }^{3}$ CSIR-Meraka, Council for Scientific and Industrial Research, South Africa \\ ${ }^{4}$ Nelson Mandela Metropolitan University, Port Elizabeth South Africa
}

\begin{abstract}
Current views of sustainable development recognize the importance of accepting the Indigenous Knowledge (IK) of rural people. However, there is an increasing technological gap between Elder IK holders and the younger generation and a persistent incompatibility between IK and the values, logics and literacies embedded, and supported by ICT. Here, we present an evaluation of new technology that might bridge generations and preserve key elements of local IK in Namibia. We describe how we applied insights, generated by ethnographic, dialogical and participatory action research, in designing a structure in which users can store, organize and retrieve user-generated videos in ways that are compatible with their knowledge system. The structure embeds videos in a scenario-based 3D visualization of a rural village. It accounts for some of the ways this rural community manages information, socially, spatially and temporally and provides users with a recognizable 3D simulated environment in which to re-contextualize de-contextualized video clips. Our formative in situ evaluation of a prototype suggests the visualization is legible to community members, provokes participation in design discussions, offers opportunities for local appropriation and may facilitate knowledge sharing between IK holders and more youthful IK assimilators. Simultaneously differing interpretations of scenarios and modeled objects reveal the limitations of our modeling decisions and raises various questions regarding graphic design details and regional transferability.
\end{abstract}

Keywords: 3D visualization, indigenous knowledge, rural, Africa, design.

\section{Partiality in Technology Design}

Information Communication Technology (ICT) continuously confronts new challenges in localizing design. Cross-cultural studies reveal Western biases in design and erroneous assumptions about the universality of concepts, methods, theories and models have led to many inappropriate decisions [1,2]. But acknowledging cultural differences between users and developers is only the start of a long and demanding design expedition when it comes to building systems to support and extend the local Indigenous Knowledge (IK) of rural people. Beyond social, economic, political and 
technological disparities between urban and rural, Western and Indigenous people lie deep tensions between the epistemologies of IK and those that underlie technology design [3]. Here, we step forward in this expedition by reflecting on our recent endeavors to build an IK management system with a rural community in Namibia, a Southern African country.

\subsection{Rural-Urban Disparities}

For many generations, rural communities in Southern Africa have acquired, produced and re-produced knowledge that sustains their lives and their environments. Practices and wisdom that respond to ecological and social contexts, and are locally validated, have enabled communities to successfully husband animals; cultivate and harvest plants; and process and conserve local resources. People share such Indigenous Knowledge (IK) orally by talking, telling stories and by participating in ordinary activities and rituals. However, various changes in Southern Africa, from education over employment to transport, have disturbed the processes of information transfer and threaten the persistence of elements of IK systems. Senior community members, or Elders, die without opportunities to pass on rural practices in ways that are accessible to younger members. At the same time younger members encounter difficulties in undertaking activities that are essential for their well-being and survival and the health of their land without their Elders' supervision or advice.

Namibia's mandatory education policies mean youth from remote areas are often sent to live with relatives in town where they will remain for years and only return in their holidays [4]. This has several consequences for local IK systems. Firstly, formal education curricula and teaching practices differ significantly from the content and processes of IK. That is, knowledge is constituted within the social and ecological rhythms of daily life for the $12 \%$ - $25 \%$ of rural residents who have never been to school; but, constituted in books and classrooms according to subjects and study timetables for those attending school. Secondly, in towns youth encounter modern technology and life-styles that contrast with those in their origin villages, which have poor sanitation, no grid electricity and sparse cell-phone coverage. After graduation some people return to their villages to reassume roles in their origin communities but encounter an increasing divide [5,6]; for instance, while they have written literacy [7] other community members have a literacy about the land and they might use technology to communicate with the "outside" world while other community members communicate according to local social protocols. Thirdly, many rural-tourban migrants remain in cities for employment but save money in order to establish homes in their rural villages later on. However, ungrounded in the minutiae of rural living, a migrant's connection to rural habitat is shaped by globalization and urban power-relations; and, again, when they return with urban-generated assets, they recontextualize rural practices. Now, keeping more livestock than before; now, travelling in vehicles not by foot or on horseback; now, listening to a radio and making detours to access a signal to use their phones not listening to a storyteller around the fire [8].

It is hardly surprising, given differences between rural and urban literacies, that there are few reports about Southern African rural communities appropriating technologies to record or process their knowledge in text, electronically, graphically 
or with videos by themselves. There are, of course, many interpretations of IK recorded by outsiders, such as historical and anthropological accounts and documentary videos. However, this type of media use is not constructed within the communication patterns of local people. Indeed, initiatives to locate technologies in rural knowledge practices are generally sparse and the locale of technology production itself, sited in research labs and design studios in cities and industrialized regions, is a conduit for selective interpretations of rural life [9].

\subsection{Acknowledging an Epistemological Gap}

Various indigenous communities globally have appropriated multi-media technology to convey their local knowledge to wider audiences [8]. In doing so they respond to certain types of politics which privilege certain sets of social, technical and literary devices and establish certain design paradigms. Leveraging privileged sets can 'give' voice to marginalized peoples but, simultaneously, suppress and distort their knowledge traditions [10]. Consider how to achieve 'development' agendas people in 'underdeveloped' regions draw on formats derived from English-language journalism and project their lived world onto a 2D-plane according to the affordances of cameras in digital storytelling (e.g. [11]) or re-present a set of oral stories in hypertext. These systems for inscription evolved beyond the IK systems of communities that share their knowledge orally, by talking and participating in everyday life not by recording in print or electronically. Choices about what to record and how to represent and disseminate it are performative in producing knowledge. They are rarely domesticated into daily practice by rural communities and, thus, neglect, for instance, information residing in the performance, structure and form of oral practices or authoring relationships between teller and audience. Further, few design studies account for the situated dynamics as IK, narrative and representation entwine or the ways people create meanings with, and about, new representations continuous with their cultural values, logics and literacies and their expectations about technology.

The dominant paradigms embedded in ICT solutions re-produce urban and western values, logics and literacies and these are often incompatible with values, logics and literacies of rural African communities. Different knowledge traditions organize and interact with information differently. That is, systems (from chronologies, taxonomies, and cartographies to authorship) do not merely translate knowledge between vocabularies but manifest a community's priorities and assumptions about reality. They draw upon implicit or explicit "theories" which encompass the kinds of relations and dependencies that do, or can, exist and their conditions of existence [12]. For instance, mainstream databases and representation and retrieval systems encode relations inherited from science and certain languages, such as hierarchies and tenses. These relations perpetuate particular perspectives on knowledge, whether that be through the structures embedded, ubiquitously, in computer filing systems to those that represent kin relations in family-trees (e.g. in Facebook) or construct the world visually from an external Point-of-view (e.g. Google Earth). And, through all of these, they are continuously shaped by writing traditions.

Dilemmas in designing technologies and media to serve marginalized knowledge traditions are not about whether local knowledge remains superficially the same but what values, logics and literacies are lost in transformation. Consider an Indigenous 
Australian Elder's disappointment with a GPS-system, which was designed to preserve his clan's knowledge on fire management but did not support the actions involved in "walking country" [8]. Consider also how a usability evaluation revealed that a sophisticated decision support system, based on ecological models, neglected the way that Herero farmers often draw upon their lived familiarity with their kinship in determining their trust of recommendations [13]. Over the three years of endeavors with the community mentioned here we have experienced similar incompatibilities between prototype technologies and members' information behavior. For instance, the rural Herero community was unenthusiastic about our attempt to use meta-data, extracted from their accounts, and printed text keywords to organize and retrieve video clips that they had collected [14]. Thus, we seek technologies that better align and reconcile with non-Western episteme and alternative approaches to design for the ways the community normally communicates about knowledge.

\subsection{Can Visualization Bridge the Gaps?}

Studies on the use of a GUI by rural communities with strong oral traditions suggest that members can more easily identify cultural icons and visualizations than using text-based technologies $[15,16]$. Visualizations of culture have a history from the earliest humans (e.g. cave paintings) which suggests that modern visualizations, which go beyond graphic icons, offer opportunities to organize information in ways that are compatible with rural IK systems. For instance, consider how Native Americans explain current situations by drawing upon a collection of stories in which events always relate to places [17] and, then, consider how a 3-D visualization of the places may offer an organizational structure that is compatible with the information conveyed in those stories. However, as noted in previous sections, for such visualizations to support the practices of IK we must account for the fundamental concepts on which they are built and the ways that community members interact with each other through the visualizations.

While modern visualization tools can combine a plethora of photo-real, surreal and abstract element in diverse visual realities the compatibility of these elements and combinations with IK is only as good as their designer's understandings about local concepts. Any visualization of a place represents a selective set of abstractions, including logics about location and time and these are by no means universal [17]. For instance, consider how 3D visualizations that separate geographical locations from temporality inadequately depict Arawakan people's stories about their journeys in Brazil [10]. Thus, producing a visualization of a place, such as a rural African village, requires compatibility with the local concepts about location and time.

Over the past decade a variety of visualizations have been created to depict IK. For instance,[18], amongst others, report on an elaborate 3D geospatial representation built for traditional custodians of the land to tell their stories by allowing users to step, virtually, into the Aboriginal dream world. However, this visualization is mediated by design teams and lacks facilities for Aboriginal people to add their own stories [19]. Further, many evaluations of visualizations designed to assist communities assume that a visualization will be experienced by a user alone, rather than be drawn into oral exchanges between several co-present users.

Here, we describe a prototype visualization of a rural village which aims to enable community members to organize information about local practices and wisdom. Our 
design draws on detailed analyses of our extensive observations of these people's spatial and temporal logics and literacies and interactions with each other and with media $[20,12,21]$. We begin by summarizing some of the ways our analysis informed designing the prototype. Then we describe how a 3D scenario-based visualization might enable local community members to upload, organize and retrieve their own video recordings of local stories and practices. Next, we present results from a formative evaluation of the visualization with the community; and, finally, we note insights on challenges of cross-cultural scenario-based visualization design that emerged in this endeavor.

\section{Places and Representation}

We developed the 3D visualization prototype as part of a long-term research programme which aims to implement Indigenous Knowledge Management systems to sustain the content, structure and communication of the IK of rural people of the Herero tribe. We chose a village in the Omaheke region in Eastern Namibia as the site for exploring the visualization because we can engage with this community continuously. The village consists of approximately 20 homesteads, each housing about seven people. The Herero include around 240.000 people living in Botswana and Angola as well as Namibia, where they are most numerous and constitute around $9 \%$ of the population. Our dialogical and participatory action research approach aims to involve community members in coevolving the design space and exploring how multi-media technology might serve their knowledge system [21]. Thus, over the past three years, we have together acquired valuable design knowledge by mutual learning and discovery. We have undertaken a range of research activities including ethnographic observations, contextual interviews, participatory design sessions, technology probes and prototype evaluations. During this process we collected some 50 video clips, some recorded by researchers and some by community members. These videos include members telling stories, describing scenarios, demonstrating local practices and engaged in everyday activities. We have interpreted and reflected upon the videos with community members in various ways and also applied Grounded Theory to analyze their content independently [12,20]. In the following sections we summarize some of our insights on how this rural community manages information, socially, spatially and temporally which has implications for designing the visualization.

\subsection{Social Significance of Places and Knowledge}

Residents in the village identify with social elements of place and refer to locations almost exclusively in terms of social relationships [12]. They build their kin relations into the physical infrastructure (since they construct their homesteads themselves) and linking places to people seems to be a feature of Herero oral traditions and their Otjiherero language. For instance, praise-names or verses describe and pay tribute to the places Herero society inhabited, before the German conquest, and have suggested cartography of the landscape in relation to people or events [22]. It appears that a familiarity with social relations makes the environment legible. For instance, locations in around villages are neither named nor signposted, although a vehicle 
registration plate marks some homesteads. We also found that places, flora and other features are intelligible, and experienced, through their associations with social roles and daily activities. For instance, activities are gendered so the fire is a place for men to eat and talk but for women to prepare food.

Villagers also described their wisdom in terms of social relationships. They communicated about their knowledge using real or metaphoric or prototypical examples and these always included relationships between people or between people and artifacts or settings. Residents frequently personalized information for the listener, explaining that "When you are telling a story with the intention of teaching you would want specific people to listen" and speakers judge the relevancy of information according to a listener's social roles. Conversely, people indicated that they trusted the integrity of information in relation to recognizing the speaker's pedigree. Further, those involved in recording video insisted that all clips should bear the village and participants' names.

Determining the relevancy of information to a listener and a knowledge-holder's pedigree involves a deep acquaintance with an intricate web of trans-generational, kin relations [20]. These trans-generational relations are reproduced in interactions with the environment. For instance, consider links between generations performed with respect to the "holy fire". This feature in some homesteads ritualizes Herero values about their society's coherence and respect for ancestors' in giving life and guarding descendants. Men use the fire in rites (e.g. ceremonial slaughtering), healing and appealing to ancestors to address social tensions. The fire has distinctive locational characteristics, sited between the house and cattle corral and separated by stones or a hedge but these vary between homesteads. The fire has a vital temporal dimension because men must ensure it is burning continuously to maintain favor with patrilineal ancestors, who may cause misfortune if displeased.

\subsection{First Person Point-of-View}

Community members refer to people and locations using Points-of-view (POV) that are intrinsic, rather than extrinsic, to the world. This was very clear when we asked participants to arrange thumbnail images spatially taken from videos they made [12]. While they walked through dense bush directly to locations, they less confidently created a geospatially accurate, aerial view despite the proximity of these locations. Community members scaled the map to the immediate area of the homestead where they sat and were reluctant to extend or re-scale to include more of the village. They sorted thumbnails to isolate those they wanted to arrange on their map effortlessly, but spent more time gesticulating around the homestead and talking about people and activities in clips than mapping. That is, people related to their environment more acutely from a First-person POV. Indeed, Otjiherero often expresses the land as a continuum in which vegetation increases with distance from the speaker and a co-located listener. We propose that a POV that is intrinsic to the world may also relate to the social-relational space of the knowledge system. People often used spatial metaphors in speech when referring to relationships; such as describing people on "this side" or, "looking in opposite directions", and this might reflect that their bodies orient spatially in social relations. Certainly, community members' use of cameras to record embodied relations between the cameraman and those recorded [20,12]. 


\subsection{Spatial and Temporal Relationships}

Villagers' paths inscribe knowledge into settings as they move between and beyond homesteads and we have proposed that their movements structure their narratives [12]. Homesteads are approximately $2 \mathrm{~km}$ apart, separated by communally used pasture, and consist of huts within a fenced yard accessed by several gates and corrals. People undertake daily activities on foot or, occasionally, on horseback, and their movements often reflect the role of animals.

For instance, men maintain camps to distribute cattle over large tracts of pasturage and thus their movement between camps invests the setting with meanings. Closer to home, people can walk between their huts, the fire, on which women cook farmed animals, and the cattle corral but must pass behind the hut to slaughter goats and cook game. That is, their paths are shaped by patterns of activities and, reciprocally, features and settings are legible via daily rhythms. For instance, a familiarity with livestock and daily and seasonal rhythms enables way finding beyond villagers' permanent homesteads. When uncertain of direction villagers follow passages created by cows' paths, read the movement of cattle between rivers, villages and corrals and recognize individual cows' footprints. [12].

Often when viewing videos villagers made sense of their contents by drawing on oral cues that referenced to seasonality, movement and sequence. Our analysis suggests that sequencing events, through movement, might enable remembering and recollecting personal and collective history, as villagers sometimes structured relationships between specific features in locations, people, livestock, and events within journeys. We also observed that community members recounted knowledge using spatio-temporal references and sequencing to order events and objects coherently. For example, one Elder referred, spatially, to a fruiting plant growing behind the homestead, before telling how to clean, cut and put its root in a calabash to sour milk and also noted learning about a novel herb: "I went to a second place in the village and was looking after the goats that got lost". [12]. That is, villagers accumulate knowledge within sets of everyday activities not by assembling separate observations at discrete times and locations.

\subsection{Re-Creating Context}

As noted in the previous section we found that recording relationships between bodies, artifacts and settings is often difficult and this constrains the knowledge a video can represent. Community members often indicated that the videos incompletely depicted knowledge. For instance, in watching a clip which shows, visually, the side on which a cow is milked, Elder-1 said it lacked information to explain "From what side, left or right?'. Settings were not always legible and villagers often found it easier to identify features from spoken sequences instead. Thus, while video records verbal descriptions, bodily actions and camera-use it excludes much information by projecting the world onto a 2D-plane. Further, the action of recording interrupts social practices in information transfer. Indeed, one Elder reflected later that he should have interviewed more actively than he would have done in an ordinary exchange so that subjects would "explain to the video" what they were doing. Viewing clips provoked community members to note that they 
needed to record more detailed explanations [20] or they added their own stories and more situational information to enrich the context for understanding. Villagers preferred to view video as a group and their exchanges about the videos further indicated the role of layering context orally to share their understandings.

\section{Scenario-Based 3DVisualization Prototype}

Our aim in creating a 3D visualization prototype is to produce a structure in which users can store, organize and retrieve an expanding corpus of user-generated videos in ways that are compatible with their knowledge system. The structure should enable rural Elders, who are considered by community members to be knowledge holders to transfer information, asynchronously, to rural-to-urban migrants, who we consider to be knowledge assimilators. To ensure that the information structure is compatible with the rural knowledge system it must enable Elders who may be textually illiterate and have little access to, and experience of, technology to store and organize videos in ways that persist important elements of social contexts.

We use various mechanisms to afford elements of social context. Firstly, we embed videos in a recognizable 3D visualization of highly familiar features of the users' village and populate this with 'generic' models of people. We position videos at locations representing those where the video was filmed and propose that users' familiarity with places in the village means that they will interpret represented locations within a social-relational space. For instance, knowing particular gender protocols associated with locations makes activities in that location intelligible and can guide a user's search for videos about those activities. Secondly, beyond graphically modeling the village we create scenarios at the locations at which videos are embedded. The scenarios are animated models of people associated with an audio narrative and we propose that by simulating aspects of communication patterns these provide resources for users to make appropriate connections in their social-relational space and restrict multiple interpretations. Thirdly, we did not prescribe paths between scenarios but rather modeled some of the paths that farmed animals follow through the village to provide some of the patterns that villagers use to navigate their setting. In the final system, Elders will position new videos at the appropriate locations in the $3 \mathrm{D}$ environment for the younger rural-to-urban migrants to assimilate the content within the appropriate context.

The current prototype was conceptualized and built by two new members of the project team (Authors 1 and 4) during their first visit to Namibia in collaboration with researchers involved in the research programme from its inception, including a Herero man from the village (Author 6). We modeled the village from Author 6's specifications and by using reference photos and videos depicting specific landmarks [23], including Google Maps satellite images. We created the visualization using the Unity 3D game engine. The visualization consists mostly of trees and bushes and a few man-made objects, such as houses, fences, water-pump, fire places and car tracks (see Figure 1) which marked specific locations as we saw on photos and videos. We populated the static environment with animals and people. 


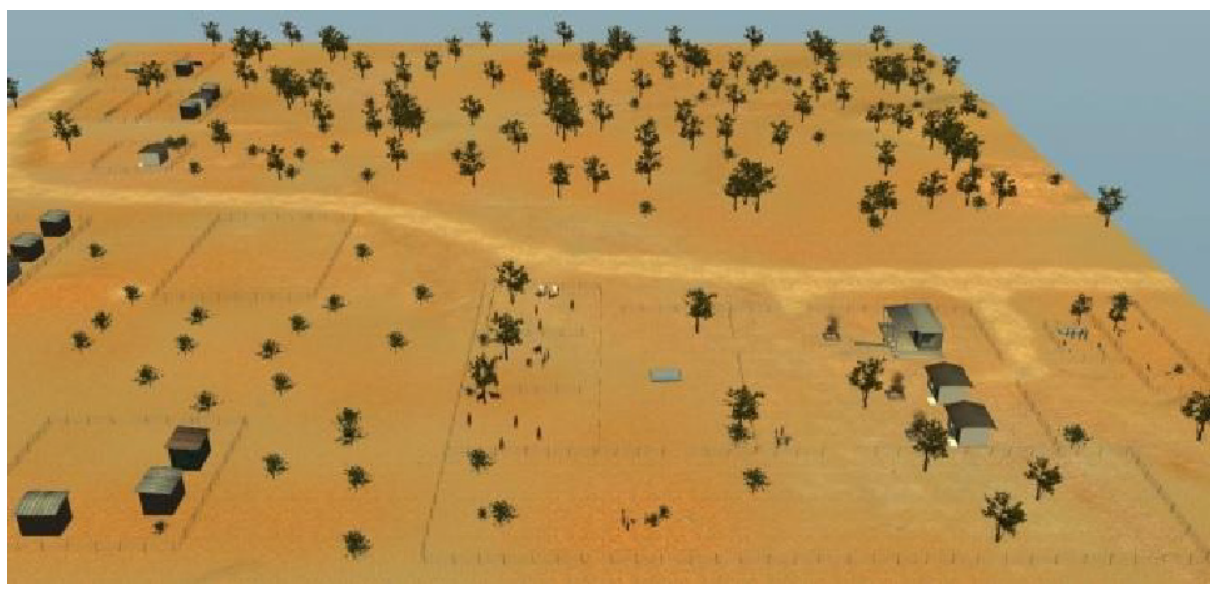

Fig. 1. The visualization including all the $3 \mathrm{D}$ objects constructed from references in the village

\subsection{Resemblance and Animation}

We focused on graphical resemblance of objects in the village and geographical positioning to support community members' recognition of locations. We assumed that community members were more likely to recognize homesteads if we modeled huts with high photo-realism, matching colors and shape, and including details like flowers (e.g. see Figure 2). We invested considerable effort in modeling goats and cows as both our ethnography and participants' frequent choice in activities they recorded suggested they are a focus of local knowledge. We attempted to model not only the forms of these animals but also their behavior by animating their bodily movements and simulating the paths they routinely follow through the village. We also modeled more nuanced details, such as smoke from a burning fire.

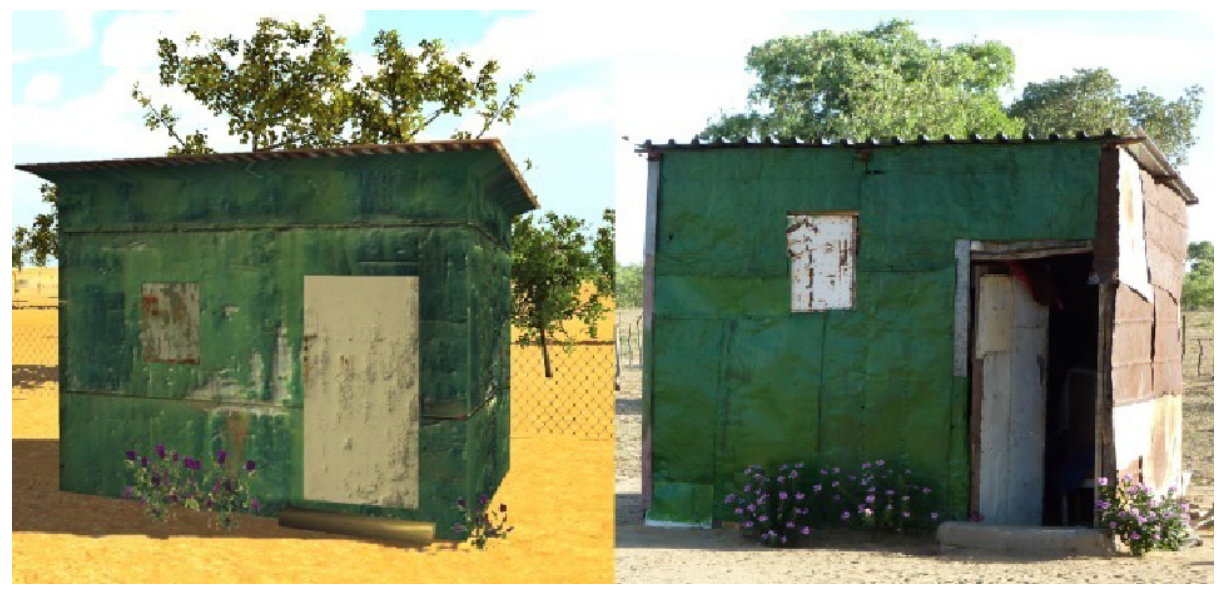

Fig. 2. The house on the left is a $3 D$ visualization of the right 


\subsection{Scenarios and Embedded Videos}

Scenarios, linking locations in the village to social activities and communication protocols, act as triggers to launch corresponding videos. The videos record community members telling stories, demonstrating everyday practices or engaged in ordinary rural activities. Community members, or various members of the team, recorded these videos over the past three years and community members have reflected on and interpreted all the videos in various ways. Videos are executed on a $2 \mathrm{D}$ plane in the visualization (Figure 3). We selected scenarios based on the extensive amount of video depicting these activities. We incorporated video in five different scenarios, which encompassed tending or slaughtering goats, healing a calf, and milking or branding cows. Scenarios respond to some of the ways the local knowledge system contextualizes information in social relationships and particular communication patterns. Each scenario consists of an audio description, recorded by a community member and models of four or five men grouped around the animal of concern. We designed multiple copies of a generic man but each instance of a man behaves a little differently. We modeled the men's gestures to suggest they are pointing and indicating that a story is being told or actions that are imminent at that location. By clicking on the area where the men are gesturing, the user is presented with the recorded video playing on a $2 \mathrm{D}$ plane hovering over the area.

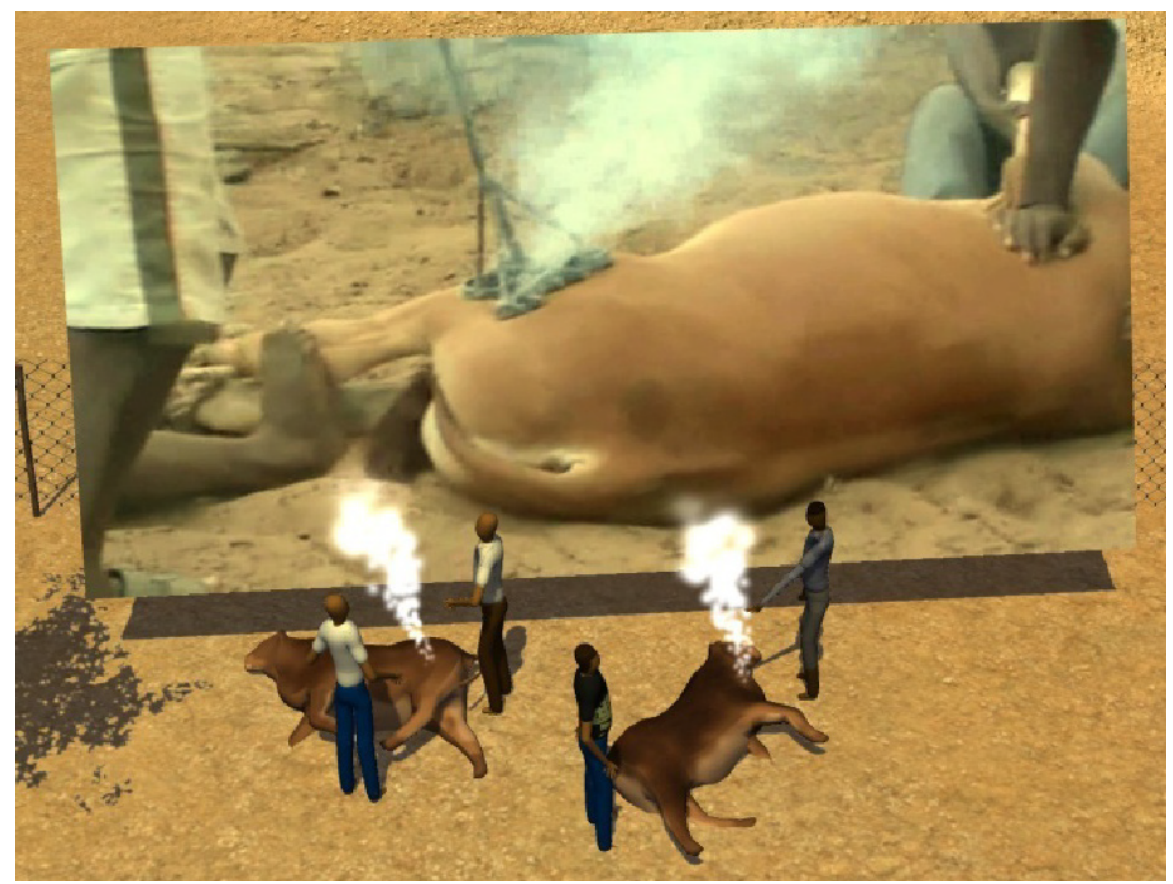

Fig. 3. Scenarios act as triggers to launch a video as a $2 \mathrm{D}$ plane in the visualization. Here the scenario includes Elders transferring information on branding cows and maintaining the herd. 


\subsection{D Placement and Navigation}

Users navigate around the village to the different scenarios by using drag-able mouse interaction that controls the camera as a lifted First Person POV. The camera has a tilted angle to provide users with a better view of social context without losing $1 \mathrm{st}$ POV. We also carefully positioned certain important objects relative; such as the holy fire, relative to the house and the kraal and the appropriate place for slaughtering.

\section{Evaluation}

We explored community members' responses to our 3D scenario-based visualization tool during a three-day trip to their village by Authors 1, 4 and 6 and another member of our project team. We sought to discuss the system with residents and evaluate how accessible the system was for them. More specifically, we wanted to determine whether or not people recognized the graphical representation of their locale and could understand that the behaviors of the people models, within a depicted scenario, connected a location to activities and triggered narrative events. We also sought to gain insight into Elders' opinions about the affordances of the scenario-based visualization and the associated embedded video in knowledge sharing and whether young people's use of the system might enable them to learn rural wisdom and skills. We scheduled two separate evaluation sessions with Elder residents of the village and younger rural-to-urban migrants who had returned to their village for the Christmas holidays. In both sessions we charged the laptop with a car battery as the village has neither electrical power nor cellphone reception.

\subsection{Community-Based Evaluation}

We pursued a community-based evaluation as our studies show the high collaboration in villagers' knowledge practices [20,12,21]. As with all our work in the village, evaluation activities were spoken in Otjiherero (villagers' native language) and were facilitated by the researcher originating from the village (Author 6). Another Otjiherero-speaking researcher also translated to provide updates on the flow of conversation to the developers (Authors 1 and 4) who cannot speak Otjiherero. We recorded the entire sessions for post-situ analysis with one static camera directed at participants and one free-floating camera. We (Author 6) translated the recorded videos in Windhoek and an external person translated and transcribed the recordings to ensure comprehensive documentation.

We framed the evaluation with four main questions. Firstly, we sought to determine if participants recognized that the visualization represented their village and could identify the site of the evaluation within the visualization. Secondly, could participants understand the scenarios in the visualization and relate these scenarios to the activities they represent; for instance, that men gather around a cow that is being milked. Thirdly, did participants understand that the people positioned in the scenarios were event triggers for the video, and did this logic make sense? Finally, we sought participants' specific ideas or reflections on the visualization. 


\subsection{System Walkthrough with the Youth}

Seven younger people, aged 10 to 18 years, agreed to participate in evaluating the system. All live in Windhoek most of the time but return to the village for holidays. The session lasted for 28 minutes and commence outside one of the newer homes in the village [see Figure 4]. We encountered some dilemmas that were due to the setup. While amiable participants were hesitant and stood too far from the laptop to see the application in the sunlight but, when we suggested moving into a house, we were reminded that people never sit and talk inside homes. After a little negotiation participants moved closer and were able to see the visualization; however, they remained reluctant to share their thoughts and during the evaluation only two, younger boys, interacted with the system. Thus, we focused the evaluation on whether participants understood the 3D model and basics concepts about its use.

We began by exploring participants' familiarity with computer use and introducing the aim of the system. Some said they have never used a computer before and others said they used computers only in schools in Windhoek. We broached the topic of sharing local knowledge by asking if participants knew what to do if a goat or cow is sick. A youth said: "We never heard a story for that" so we (Author 6) pursued this by saying that the Elders are the holders of this knowledge and asking "if the Elderly die, what happens with this knowledge?". The youth agreed that this knowledge "will die with them as well." .

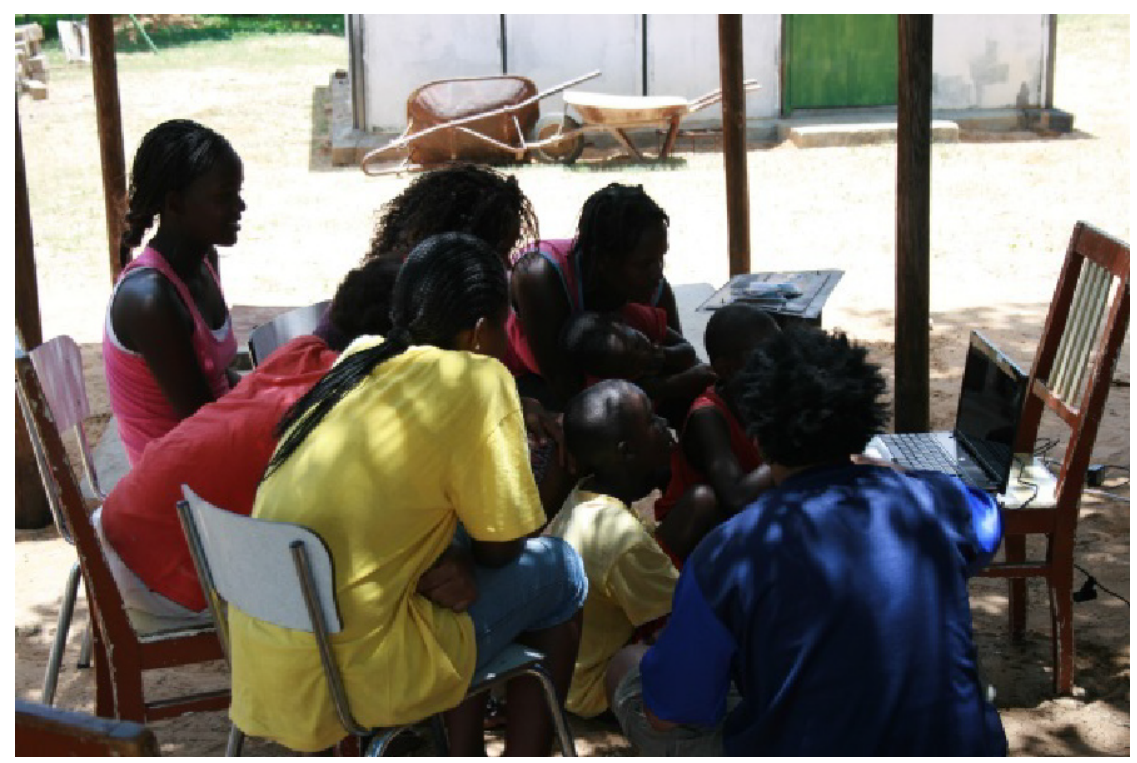

Fig. 4. We demonstrated the system to seven younger rural-to-urban migrants

After their initial shyness the young people began to comment on the system and about what they saw. These suggest that they connected the visualization to their imagery of various objects (or perhaps pictures of objects). For instance, exclaiming 
"People! Fire! Houses and trees!" We found that participants were able to navigate and 'read' the visualization and interpret objects using a tilted camera perspective. For instance, some of the youth recognized the $3 \mathrm{D}$ representation of the house outside which we conducted our evaluation: "This is here. It is this house." Afterwards, they explained that they recognized it from the sides of the house and the fires in front of it. When participants panned over the cattle-branding scenario they mentioned, immediately, the branding irons, smoke and cattle, and then watched a video on branding cattle. Thus, we asked if the scenario was a story and they replied that the cattle were in the kraal (corral) and that because a man was pointing this indicated that he (the model) was telling a story. Another interesting discussion ensued after a youth panned over a scenario about slaughtering a goat. To begin with participants vacillated: "It's a goat. It's a sheep." but then suddenly agreed that sheep do not have small tails. We interpret this ambiguity as indicating both the importance of photorealistic quality and, that the users' and developers' emphasis on specific visual details differs. That is, we (Author 1 and 4) emphasized man-made objects but not the very fauna that make for local livelihoods and settings.

\subsection{Elders' Comments on the System}

We conducted a 24 minutes evaluation session with Elders in the shade outside of a home, and 2 village Elders and a group of 3 women aged over 35, and 3 younger people sat around the prototype. The Elders have no experience of computers and did not try to interact with the visualization, perhaps because of the set-up and composition of the group or perhaps because the group has been more used to watching videos on the laptop in our earlier work (Figure 5).

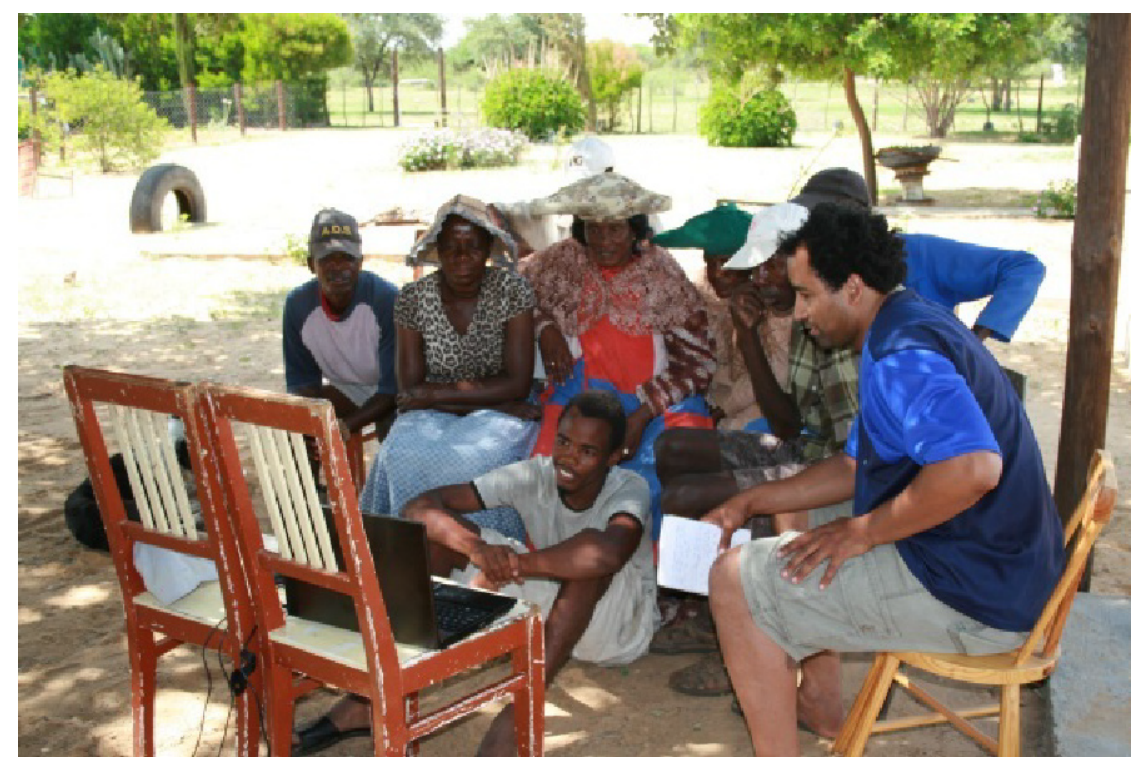

Fig. 5. We demonstrated the system to 8 rural residents including 2 Elders 
The Elders observed the resemblance between the visualization and where the evaluation occurred; however, commented on the visual accuracy of the features. For instance, when discussing the scenario near the cattle in the kraal, the Elders told us that planted trees do not grow inside the kraal and added: "It's not a planted tree, it's a wild tree (Otjindanda), just look the way it grows!" Our (the developers) unfamiliarity with local culture and environment meant we lacked an appreciation of visual differences between cultivated and wild trees or where they might grow and made many assumptions when we drew upon reference images. Our lack of visually differentiating trees made the setting less legible to the Elders. This indicates that they not only understand the visualized objects but also recognized the importance of placing objects. Lack of visual accuracy sometimes caused the Elders' frustration and uncertainty about their own memory of the setting; for instance, based on photos we modeled one block of concrete, where villagers lay their fire, in front of a house. However, this hut has two blocks of concrete.

The Elders also noticed inaccuracies in the behaviors of modeled people. For instance, they understood the scenario about milking the cow but said that it looked as if the person was tying the cow. They also understood the goat slaughter scenario easily but, when we asked them who was telling the story, they said that it was the model in the middle of the group because "He is pointing and showing what to be done." While this reassures us that the Elders reason about the visualized objects it also indicates the need to invest, substantially, in designing and modeling of fauna and objects to match local priorities. At the end of the session we asked for the Elders' general impressions of the visualization and they said "They are good in their look [quality] and in the sense that they will be kept there forever and they will never be forgotten." We also asked their opinion about the system's potential in teaching; they said: "Very much! Especially those township youth, they don't know village stuff, they will learn from that."

\section{Reflections and the Way Forward}

The evaluation suggests that re-contextualizing video clips in a recognizable 3D visualization of a familiar environment can potentially bridge epistemological, ruralurban, technological and generational gulfs. However, simultaneously, the evaluation shows differences between developers' and users' interpretations of visualized scenarios and objects. This leaves us with a number of challenges in the next phases of the research.

\subsection{The Importance of Realistic Details}

Both youth and Elders paid acute attention to specific details such as posture, gesture, animal features and behaviors, trees, and the positions of some objects, such as fire. These details seem to contribute to recognizing places as well as to the integrity of representing IK. In evaluations participants discussed, at length, the "wrong posture" of the milking people who are supposed to kneel rather than stand. Such 
conversations are critical in designing the system. Community members clearly indicated which details are important which both re-emphasized local practices and that the details that scenarios must include in order that they enable sufficient social framing. References to specific details indicate that some items require greater accuracy but our evaluation did not reveal the importance of photo-real properties, such as the color of houses or the size of the trees. Due to our unfamiliarity with the Namibian landscape the developers (Authors 1 and 4) we emphasized photo-real resemblance for man-made objects (e.g. the communal water pump, huts), assuming that these visually differentiated settings. However, as the Elders indicated, we neglected important features and distinctions between trees. This shows the vital importance of gathering data about people's interactions with items in their environment, linguistically and visually, and translating this in the visualization.

\subsection{Interpretations and the Need for Community-Driven Design}

Interpreting visual representations depends on a viewer's cultural background. For example, we intended the cow milking scenario to represent people milking cows and others watching. However, the posture of those milking and the gestures of those watching signified to community members that the scenario was about teaching milking. Drawing upon information sources such as photographs, videos and a community member's description was a practical step in creating the visualizations; but, as indicated by rich ethnographic depictions yielded by extensive stays in the village $[12,20]$ they cannot match direct and collaborative engagement. This resonates with observations in various earlier activities about differences in visual recognition between community members and ourselves and community members comments that video incompletely depicts visual details. Thus, our next design steps are situated in the village developing the visualization with the community members. These prolonged situated development periods will enable us to explore how to design the visualization to respond to the ways community members movements structure their narratives. Like various other visualization endeavors with indigenous groups [18] our current prototype allows users to explore the setting without forcing a linear narrative between scenarios. However, this might compromise the information contained in the sequences of movement that contextualize knowledge [12]. A situated approach to development not only provides continuous integrity checking but also insight into the dynamics of situated interactions between local IK and the visualization. Our previous analysis of community members' interactions with video showed the narrative structure of people's comments and stories changed when local people viewed videos [12,20]. Such insights are important to avoid making design decisions that will suppress and distort local knowledge [10].

\subsection{Further Features and Transferability}

Our further development includes focusing on appropriate scenario design and modeling the clothing, posture, gestures and movements of people. We will also explore time-location-activity relations as our earlier, and ongoing, ethnography repeatedly shows the importance of daily rhythms to navigating the village and coordinating activities. We can realize shade movements easily in the game engine and this will enable varying scenarios at locations according to time of day. 
We will also layer audio of community members' different interpretations of video clips as our previous studies reveal ambiguity decreases or increases when members add stories and information [20]. Currently, we propose that users click on one of the people in the scenario people to access perspectives. Such a feature might also contribute to personalizing information according to the user; however, we also need to explore differential access to scenarios such that users interact with scenarios based on gender, kinship, age or other criteria [12]. Access to different layers is increasingly used for various visualization and mapping projects for Australian indigenous people, where the suitability of information transfer depends on place, age, clan and gender [8].

Investing in the specific details of a specific village has consequences for the transferability of the application to other villages. Our current prototype serves as a proof of concept and we are eager to explore which features of the visualization can be re-used for other villages and for other regions. We will explore this by running further evaluations in other sites in Namibia.

\section{Conclusion}

Designing an appropriate visualization to support IK in rural Africa requires thoroughly understanding situated interactions between knowledge systems and audio-visual representations and the conceptual frameworks bearing upon design. That is, to design digital infrastructures for currently unserved IK we must account for the transformations that take place as technology interacts with the lived experiences, actions and thought or spoken narratives that constitute knowledge [20].

We have shown the potential of embedding video recorded by, or with, communities within a scenario-based visualization to bridging some of the epistemological, generational, literacies and technological gulfs in Eastern Namibia. We claim that the prototype can help to re-instantiate IK by supporting information sharing. Embedding videos in scenario-based 3D visualization is a first attempt to re-contextualize decontextualized representations in a locally accessible way. Merging information about activities and locations visually appears to be more intuitive for this African rural community than our earlier attempts with text-based and video-only retrieval. Our formative evaluation of the prototype in Namibia indicates that both Elders and younger people relate to the representation and can participate in discussions about improving and appropriating the system. We continue to explore some of the challenges that further development entails, such as the degree of detail, scope of interpretations and transferability between communities. However, we hope our reflections on this novel structure for organizing video, according to a community's familiarity and priorities, will stimulate international dialogue on the contribution that scenario-based 3D visualizations to other local knowledge systems.

Acknowledgements. We thank the residents of the village in Namibia for evaluating our prototype and their continuous commitment and inspirational participation in the project. The work described in this paper was partly funded by Det Obelske Familiefond. 


\section{References}

1. Winschiers, H., Fendler, J.: Assumptions Considered Harmful. In: Aykin, N. (ed.) HCII 2007. LNCS, vol. 4559, pp. 452-461. Springer, Heidelberg (2007)

2. Evers, V., Hinds, P.: The Truth about Universal Design: How knowledge on basic human functions, used to inform design, differs across cultures. In: Proceedings of the 9th International Workshop on Internationalisation of Products and Systems, Building Global Design Communities (July 2010)

3. Bidwell, N.J., Browning, D.: Pursuing Genius Loci: Interaction Design And Natural Places. Pers. Ubiq. Comp. 217 (2009)

4. Rumble, G., Koul, B.N.: Open Schooling for Secondary and Higher Secondary Education: Costs and Effectiveness in India and Namibia. Vancouver, Commonwealth of Learning, http: / /www.col.org/resources/publications / consultancies / Pages / $2007-07$ S (accessed: November 2010)

5. Chinn, M.D., Fairlie, R.W.: The Determinants of the Global Digital Divide A CrossCountry Analysis of Computer and Internet Penetration. Working Papers 881, Economic Growth Center, Yale University (2004)

6. Fuchs, C., Horak, E.: Africa and the digital divide. Telemat. Inf. 25, 99-116 (2008)

7. UNESCO, International Literacy Statistics: A review of Concepts, Methodology and Current Data. Institute for Statistics, Montreal (2008),

http: / / www .uis. unesco.org

8. Bidwell, N.J., Standley, P., George, T., Steffensen, V.: The Landscape's Apprentice: Lessons for Design from Grounding Documentary. In: Proc. Designing Interactive Systems (DIS), pp. 271-280. ACM Pr., New York (2008)

9. Bidwell, N.J., Browning, D.: Pursuing Genius Loci: Interaction Design And Natural Places. Pers. Ubiq. Comp. 217 (2009)

10. Green, L.J.F.: Cultural heritage, archives \& citizenship: reflections on using Virtual Reality for presenting knowledge diversity in the public sphere. Critical Arts 2(21), 308320 (2007)

11. Taachi, J., Kirran, J.: Finding a voice: Themes \& discussion. Technical report, UNESCO (2008)

12. Bidwell, N.J., Winschiers-Theophilus, H., Koch Kapuire, G., Rehm, M.: Pushing personhood into place: Situating media in rural knowledge in Africa. International Journal of Human-Computer Studies (2011)

13. Winschiers, H., Fendler, J., Stanley, C., Joubert, D., Zimmermann, I., Mukumbira, S.: A Bush Encroachment Decision Support System's Metamorphosis. In: Proceedings of the 20th Australasian Conference on Computer-Human Interaction: Designing for Habitus and Habitat, Cairns, pp. 287-290. ACM, New York (2008)

14. Kapuire, G., Winschiers-Theophilus, H., Blake, E., Bidwell, N., Chivuno-Kuria, S.: A revolution in ICT, the last hope for African Rural Communities' technology appropriation. In: IDIA 2010, Cape Town (2010)

15. Parikh, T., Ghosh, K., Chavan, A.: Design studies for a financial management system for micro-credit groups in rural India. SIGCAPH Comput. Phys. Handicap. 73-74, 15-22 (2002)

16. Kuicheu, N.C., Fotso, L.P., Siewe, F.: Iconic communication system by XML language: (SCILX). In: Proceedings of the 2007 International Cross-Disciplinary Conference on Web Accessibility (W4A) (W4A 2007), pp. 112-115. ACM, New York (2007)

17. Brewer, J., Dourish, P.: Storied spaces: Cultural accounts of mobility, technology, and environmental knowing. Int. J of Human-Computer Studies 66(12), 963-976 (2008) 
18. Pumpa, M., Wyeld, T.G.: Database and Narratological Representation of Australian Aboriginal Knowledge as Information Visualisation using a Game Engine. In: Proceedings of the Conference on Information Visualization (IV 2006), pp. 237-244. IEEE Computer Society, Washington, DC, USA (2006)

19. Turner, J., Browning, D., Bidwell, N.J.: Wanderer Beyond Gameworlds Leonardo Electronic Almanac 16(2-3), Part 1 Embodiment and Presence (2009)

20. Bidwell, N.J., Winschiers-Theophilus, H., Koch Kapuire, G., Shilumbe Chivuno-Kuria, S.: Exploring Situated Interactions Between Audiovisual Media \& African Herbal Lore. Personal and Ubiquitous Computing (2010)

21. Winschiers, H., Bidwell, N.J., Blake, E., Chivuno-Kuria, S., Kapuire Koch, G.: Being Participated - A community approach. In: Proceedings of the 11th Participatory Design Conference, Sydney (November/December 2010)

22. Bubenzer, O., Bollig, M., Kavari, J., Bleckmann, L.: Otjiherero praises of places collective memory embedded in landscape and the aesthetic sense of a pastoral. People Studies in Human Ecology \& Adaptation 4, 473-500 (2009)

23. Haasbroek, J.L.: The challenges of utilizing intelligent human-computer interface technology in South Africa and other African developing countries. In: IEEE International Conference on Systems, Man and Cybernetics, Chicago, vol. 1(18-21), pp. 821-826 (1992) 